\title{
Improvement in the efficiency of the distributed power systems
}

\author{
G. BENYSEK ${ }^{*}$
}

University of Zielona Góra, Institute of Electrical Engineering, 50 Podgórna St., 65-246 Zielona Góra, Poland

\begin{abstract}
It is common knowledge that transmission systems are being pushed ever closer to their stability and thermal limits. Traditional solutions for upgrading electrical transmission system infrastructure have been primarily in the form of new power plants, new transmission lines, substations, and associated equipment. However, as experience has proven, the process of authorizing, locating, and constructing new transmission lines has become extremely difficult, expensive and time-consuming. This is the reason why during the last few years interest in dispersed generation, power flow controlling or/and energy storage has increased significantly - interest in solutions which can improve the efficiency of the distributed power system.

With respect to the above the major aim of this paper is to present the fundamental problems of the Power Systems and the improvement methods which arise using Distributed Generation. The paper introduces also a system for the utilization of energy generated on the customerside - this arrangement is realised using a Voltage Active Power Filter. The experimental results presented in the paper confirm the flexibility of this arrangement in efficiency improvement processes.
\end{abstract}

Key words: distributed generation; interface, efficiency.

\section{Introduction}

Today, most users are inactive receivers of electricity without further involvement in the management of the sources and the grid; each user is simply a hole for electrical energy. Additionally, much of the equipment of today's Polish Electrical Power System (EPS) was installed with a nominal design life of about 30-40 years; meanwhile, the size of the load has grown above what was predicted when the grid was designed. In response to the above and to the climate change challenge, which is forcing reduction of greenhouse gases emissions, Poland and other European countries have started the process of liberalization of their electrical power systems, opening access to grids and encouraging renewable energy sources. In this situation, there is a great opportunity for Distributed Generation (DG) and other new players to provide better network capability, flexibility and functionality - improvement in the efficiency. As a result, the amount of electrical energy produced by large conventional plants will be displaced by DGs, demand response and energy storage.

Taking into consideration the above, the major aim of this paper is to introduce the fundamental problems of Electrical Power Systems and, secondly, to review efficiency improvement services to grids provided with inverter interfaced Distributed Generation. The paper introduces also some powerelectronics based solutions, which form a basis for building single as well as multilane DG/EPS interfaces.

\section{Structure and fundamental problems of Electrical Power Systems}

Today's grids are primarily based on large power stations connected to transmission lines which supply power to dis- tribution systems, thus the overall image remains the same as ever: one-way power flow from the power stations, via the transmission and distribution systems, to the final customer (end-user).

The one-line diagram shown in Fig. 1 illustrates today's Electrical Power System and its major components: generation, transmission and distribution. Electric power is generated at power stations predominantly by synchronous generators that are mostly driven by steam or hydro turbines. Hence, the electric power generated at any such station usually has to be transmitted over a great distance, through transmission systems to distribution systems. The distribution networks distribute the energy from the transmission grid or small/local Distributed Resources (DR) to customers.

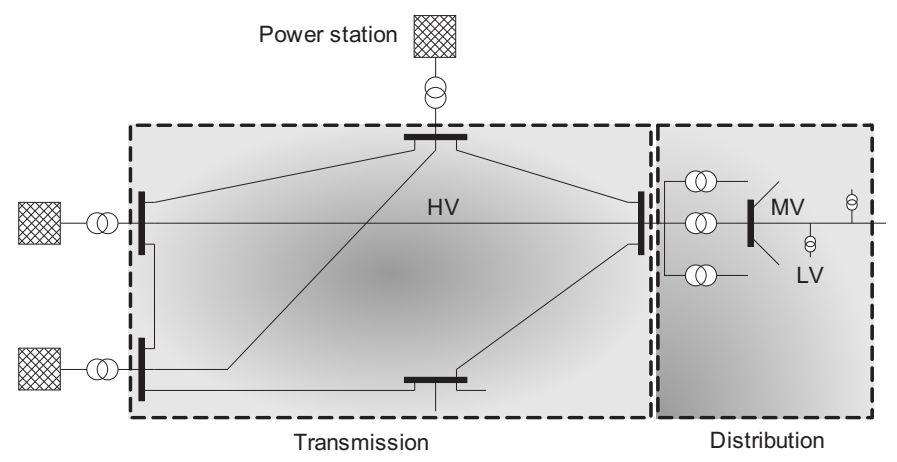

Fig. 1. A simplified one-line diagram of the power system

The three mentioned components - generation, transmission and distribution - have different influences, individual and sometimes common, on the efficiency of power system. There are many issues involved, such as the maintenance of

*e-mail: G.Benysek@iee.uz.zgora.pl 
power apparatuses and the system, the stability of the operation system, faults, distortions, loads and non-linearities etc.

2.1. Transmission systems. Transmission systems are being pushed ever closer to their stability and thermal limits and if the facilities are not suitably upgraded the power system becomes vulnerable to steady-state and transient stability problems [1-6]. In such environments, transmission capacity becomes a virtue; therefore during the last few years interest in the possibilities for controlling power flows in transmission systems, dispersed generation or energy storage has increased significantly.

Traditional solutions for upgrading electrical transmission system infrastructure have been primarily in the form of new power plants, new transmission lines, substations, and associated equipment. However, as experience has proven, the process of authorizing, locating, and constructing new transmission lines has become extremely difficult, expensive and time-consuming. It is envisaged that, alternatively, Flexible Alternating Current Transmission System (FACTS), Energy Storage Systems (ESS) and Distributed Generation can enable the same objectives to be met.

The potential benefits of employing the above-mentioned solutions include reduction of operation and transmission investment costs and implementation time compared to the construction of new transmission lines, increased system security and reliability, increased power transfer capabilities, and an overall enhancement of the quality of the electric energy delivered to customers.

2.2. Distribution systems. With the emergence of computers, sensitive loads and modern communications, a reliable electricity supply with high quality voltage has become a necessity. Voltage sags and dips can cause loss of production in automated processes, and can also force a computer system or data processing system to crash. A sustained overvoltage can cause damage to household appliances. An undervoltage has the same effect as that of voltage sag. Voltage imbalance can cause temperature rises in motors. Harmonics, DC offset, can cause waveform distortions. Unwanted current harmonics flowing across the distribution network can cause losses and heating in transformers and Electromagnetic Interference (EMI) [7]. Interharmonics voltages can upset the operation of fluorescent lamps and television receivers. They can also produce acoustic noise. It can be concluded that the lack of quality power can cause loss of production, and damage to equipment.

As with FACTS and other players in transmission systems, power electronics devices called Custom Power Systems (CUPS) together with ESS, DG and smart end-user appliances can be applied to the power distribution systems to increase reliability and quality of power supplied to customers [8-12]. Through those technologies the reliability and quality of the power delivered can be improved in terms of reduced interruptions, reduced voltage, current variations and distortions. The proper use of these technologies will benefit all industrial, commercial and domestic customers.

\section{Distributed Generation's efficiency improvement services}

As Distributed Generation hardware becomes more reliable and economically feasible, there is a trend to connect DG units to the existing utilities to serve different purposes and offer more possibilities to power system users. DG can bring some relief for Transmission and Distribution (T\&D) capacity and these new energy injections can have some benefits in the operation on the whole EPS:

- The production of energy near the load reduces the losses of the grids, because energy is generated where it is consumed.

- The existence of DG units could be an important way to increase the voltage; the insertion of a DG in a bus raises its voltage.

- End-users who place DG can benefit by having backup generation to improve reliability.

Distributed Generation hardware can also secure the power flow control actions. There are a number of reasons for this:

- In a meshed power system, there can occur a situation where a low impedance line carries much more power than originally designed for, while parallel paths are underutilized. With power flow control, the stressed line can be relieved, resulting in better overall utilization of the network.

- Both low voltage as well as high voltage can exceed the voltage limits and therefore corrective actions have to be taken. The corrective actions with utilization of selected FACTS devices include correcting the power factor and compensating reactive losses in lines by supplying reactive power.

- Transient and dynamic stability control issues-transient stability describes the ability of the power system to survive after a major disturbance, while dynamic stability describes sustained or growing power swing oscillations between generators or a group of generators initiated by a disturbance. In the first as well as the second situation, active power flow control can be a solution.

Summarizing, power flow control technologies have the abilities to solve both steady-state (better utilization of the transmission assets, minimization of losses, limit flows to contract paths etc.) and dynamic issues (dynamic dumping of the oscillations) of transmission systems.

Considering the above the only concern is to find DGs/EPS interconnection solution which is suitable to secure all above actions.

\section{Design and build of a DGs/EPS interconnection systems}

4.1. Stand-alone systems (off-line/off-grid). Usually this type of interconnection is used in the case of distribution network damage as an uninterruptible power supply. Energy produced by the generator is delivered to the load through 
the voltage source inverter, then connected to the load terminals. To the disadvantages of this solution one can add the need for reconfiguration actions (to allow energy flow from customer-side generator through the converter to the loads, the line switch has to be turned off and the converter switch has to be turned on) which leads to temporary lack of supply.

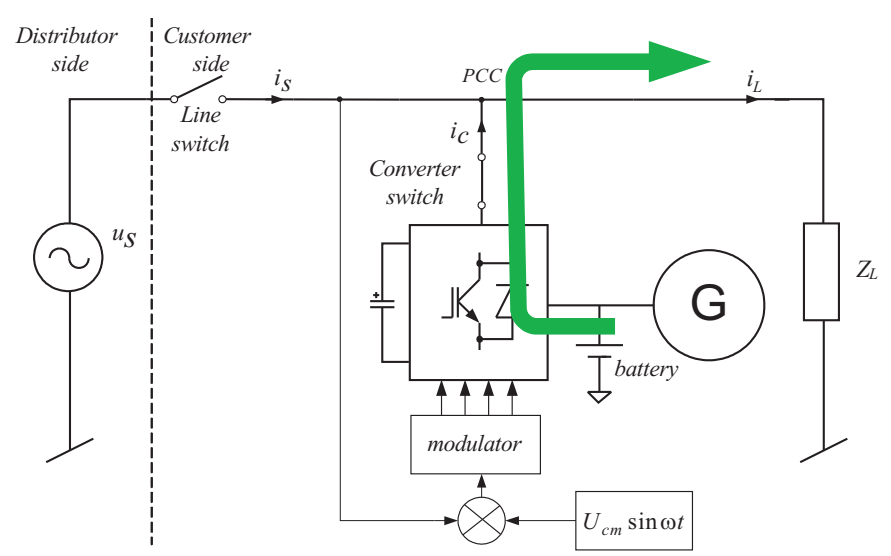

Fig. 2. Customer-side generation in stand-alone system

In this method of interconnection, it is possible to connect generators (generators with stable voltage amplitude and frequency) without the need for output voltage synchronization with the grid. Although not needing synchronization, the other load voltage parameters have to be maintained, therefore this type of energy delivery can be referred to as voltage type interconnection.

subsectionGrid-connected systems (on-line). On-line operation of AC customer-side generators connected to the grid is difficult because it requires synchronization, therefore in the case of an on-line operation requirement it is more practical to use double conversion systems, where the generator is connected through the rectifier to synchronized inverter [13, 14].

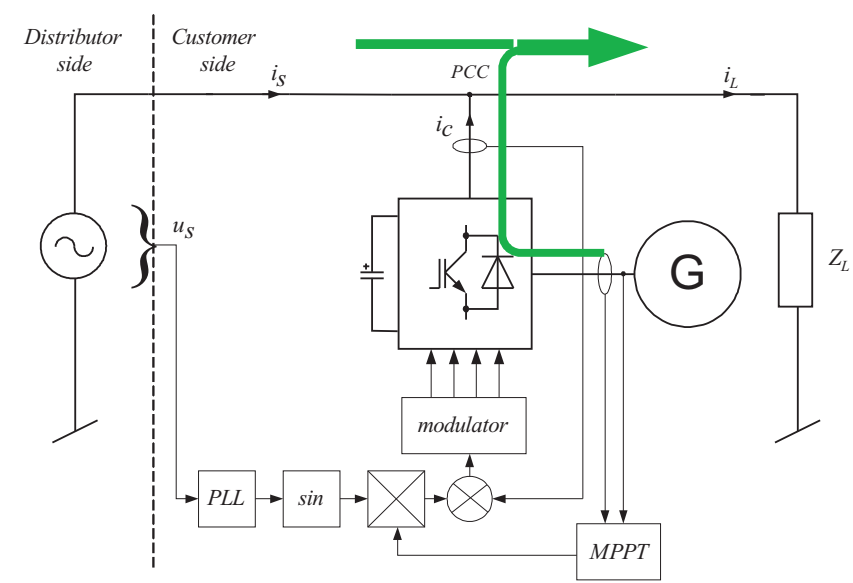

Fig. 3. Customer-side generation in grid-connected system

Energy delivered by the inverter is balanced with energy delivered through the distribution network, thus a balance at the point of common coupling $P C C$ is obtained as a result of the currents sum. In this type of solution the inverter is controlled as the parallel current source, thus this kind of interconnection is called current type.

When grid fault occurs, the inverter can secure the continuity of supply but requires changes in control strategy, otherwise load voltage depends on load power and can't be stabilized.

4.2. Voltage Active Power Filter in interconnection mode of operation. As a more powerful and flexible alternative to the above presented a Voltage Active Power Filter (VAPF) working in interconnection mode of operation can be considered [11]. In this solution the inverter is connected to the network terminals through the series reactance $X_{S}$ and then directly to the load. The whole arrangement can be broken down into three major parts: the Grid Side Inverter (GSI)(T1-T4), the DC link $\left(C_{1}\right)$ and the Generator Side Converter (GSC)(T5, T6). In the proposed solution, the grid side inverter is controlled as the voltage source, connected to the network terminals through the series reactance $X_{S}$, see Fig. 4. The arrangement generates on its output stabilized, sine wave voltage with the same parameters as the grid, but shifted with given angle $\delta$ (proportional to the load active power). The $\delta$ angle is proportional to load active power $P_{L}$ and is calculated using the power balance method, which is commonly used in FACTS devices $[11,15]$. In this method the process of the DC link capacitor voltage stabilization requires balancing of energy delivered and taken from this capacitor in each calculation period. As a result the $U_{X s}$ voltage on the series reactance $X_{S}$ is sinusoidal, thus the line current $i_{S}$ is sinusoidal as well and its shape is independent of load current $i_{L}$. Moreover, the inverter's output voltage $u_{C}$ is the same as load voltage and its magnitude $U_{C m}$ is stabilized. To summarise, the proposed solution realizes three functions: current active filtering, load voltage stabilization and, in case of grid voltage faults, an uninterruptible load supply, the last function is only possible with energy storage device.

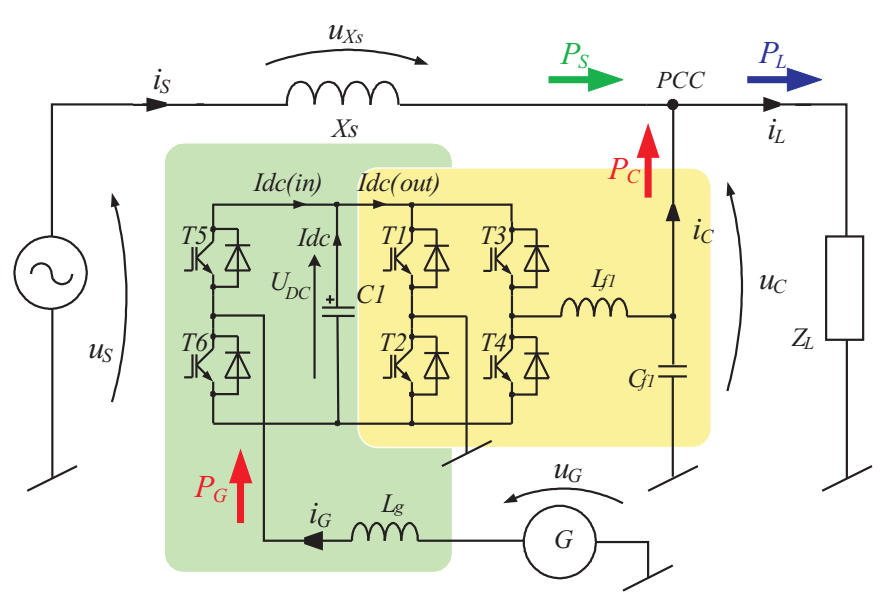

Fig. 4. VAPF as interconnection system

Additionally the grid side inverter can be a platform for a more advanced solution, which could be an interconnection of the distributed generators (AC as well as DC customer side generators) with the grid [15]. To allow the interconnection 
mode of operation, the customer side generators can be connected to the DC link, using a two-quadrant converter: the generator side converter.

There are two points of power balance in this structure. Although the first one is on the inverter's DC side and second one on its AC side, they are dependent on each other. This is so because the inverter control strategy is based on the DC link capacitor voltage stabilization, which requires balancing of the energy delivered $E_{C+}$ and taken $E_{C-}$ from the capacitor in each calculation period $T_{C}$ :

$$
\begin{gathered}
\Delta E_{C}=E_{C+}+E_{C-}=C 1\left(U_{D C(0)}^{2}-U_{D C(T c)}^{2}\right) / 2= \\
=P_{C} \cdot T_{C}=\int_{0}^{T c} p_{C}(t) d t=0,
\end{gathered}
$$

where $p_{C}(t)$ - inverter instantaneous power, $P_{C}-$ inverter average power.

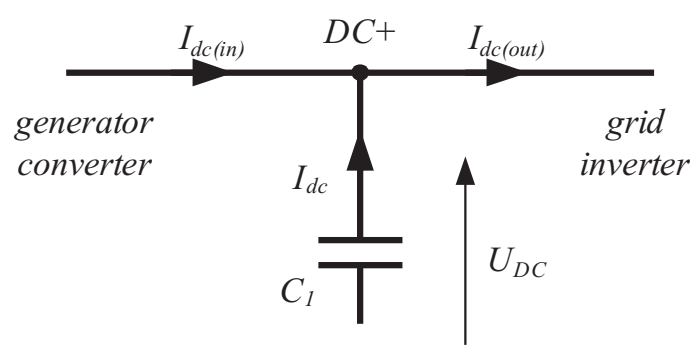

Fig. 5. DC side power balance

In every $T_{C}$ period the average currents for DC side power balance point DC+ are given by the following Kirchhoff formula, see Fig. 5:

$$
I_{d c(\text { in })}+I_{d c}=I_{d c(\text { out })}
$$

and DC link capacitor average current is defined by the equation:

$$
I_{d c}=C_{1} \cdot \Delta U_{D C} / T_{C}
$$

The DC link voltage changes $\Delta U_{D C}$ are compensated by the grid inverter control strategy and in this case the capacitor average current (3) in every balancing period $T_{C}$ is:

$$
I_{d c}=0
$$

and this means:

$$
\left.I_{d c(\text { in }}\right)=I_{d c(\text { out })} .
$$

After short considerations on power balance in $\mathrm{DC}+$ and $P C C$ points one can claim that using the proposed methodology energy delivered to the DC link from customer-side generator $G$ can be utilized through a grid inverter without any changes in control strategy. Although the operation rules as well as controller structure are the same, the AC side power balance equation should be modified:

$$
P_{L}=P_{S}+P_{C}
$$

and

$$
P_{S}=P_{L}-P_{C} \stackrel{P_{C}=P_{G}}{\longrightarrow} P_{S}=P_{L}-P_{G}
$$

In (6) and (7) powers, delivered to the $P C C$ point have to be the same as power absorbed by the load. The generator converter controls the value of this energy and plays the role of the master in this structure. In Fig. 1 transistors $T 5$ and T6 constitute a current type generator converter and energy delivered through this converter from the generator $G$ to the capacitor $C 1$ causes DC link voltage $U_{D C}$ to increase. To stabilize this voltage the grid inverter T1-T4 has to push this energy into the grid, thus there will be a power unbalance at $P C C$ point and as a result the shift angle $\delta$ should be decreased to reduce the line power $P_{S}$. This situation is repeated in every balancing period $T_{C}$. Power delivered from the grid inverter can be utilized by loads or injected to the system in situation when $P_{C}>P_{L}$. The load magnitude is kept at nominal value just as in stand-alone systems and line current is controlled to fulfil the power balance as it is in grid-connected systems, thus the proposed solution is a combination of the voltage and current type interconnection. It is worth mentioning here, even if the proposed arrangement works in interconnection mode of operation, that all earlier declared "power quality improvement capabilities" are fulfilled.

The Generator Converter. Transistors T5, T6 create a two-quadrant current type converter, they are switched opposite each other. The control strategy is determined by generator type, however for both generator types (DC as well as AC) the value of the delivered energy is determined by the generator current $i_{G}$ forced in closed loop. The value of this current is calculated in the maximum power point tracking MPPT block which allows optimal generator utilization. The main difference between DC and AC generators is in shaping of the generator current $i_{G}$ which has to be the same shape as the generated voltage. This is so because in variable speed generators there are strong dependencies between the output voltage parameters (magnitude and frequency) and speed of the shaft. Figure 6 shows proposed control strategies for both generator types.

a)

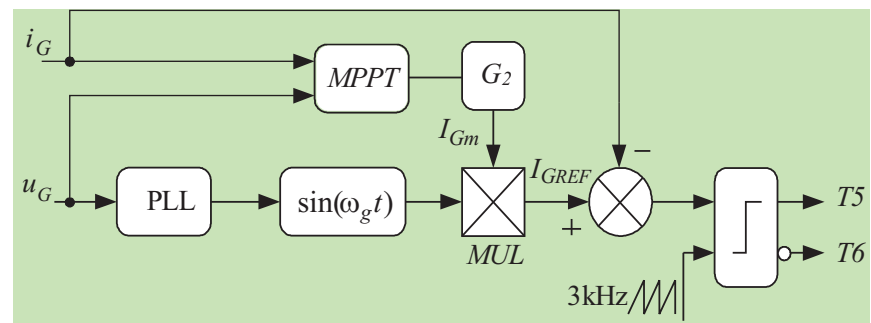

b)

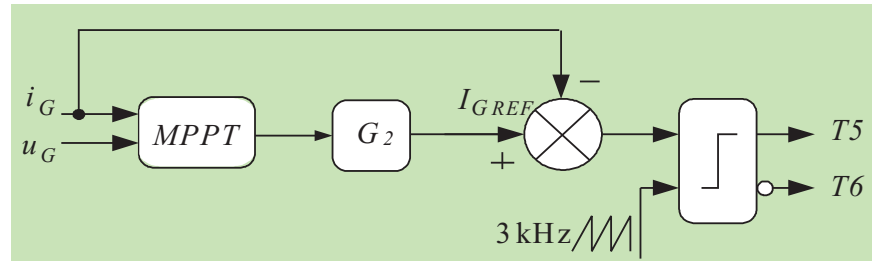

Fig. 6. Generator side controller for: (a) AC generator, (b) DC generator 


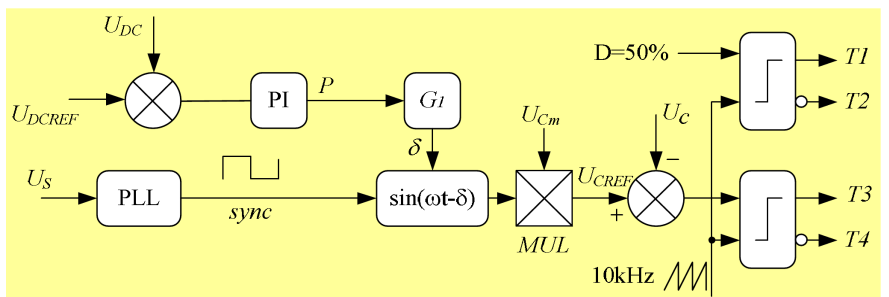

Fig. 7. Grid side inverter controller

a)

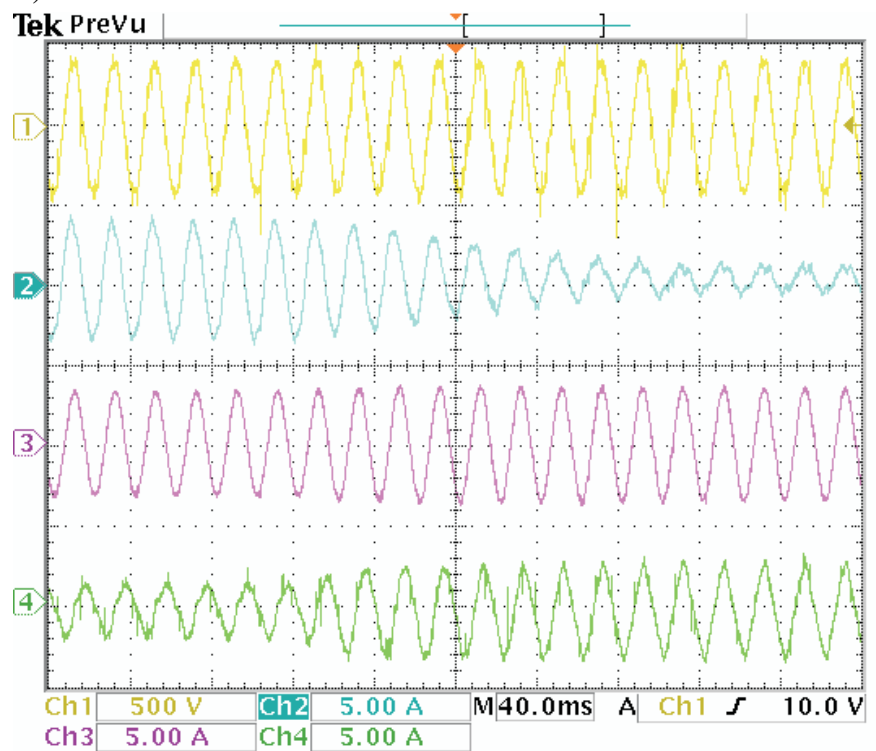

b)

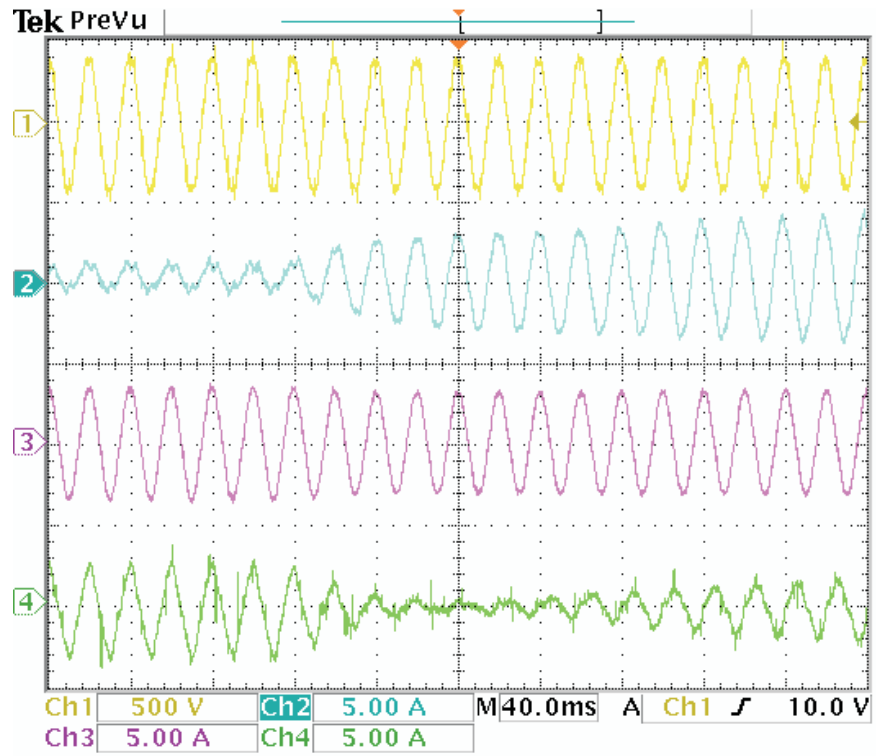

Fig. 8. Experimental results during $P_{G}$ changes ( $D C$ customer side generator; $P_{G}<P_{L}$ ): (a) increased $P_{G}$, (b) decreased $P_{G}$; (Ch1 $\left.u_{S}, \mathrm{Ch} 2-i_{S}, \mathrm{Ch} 3-i_{L}, \mathrm{Ch} 4-i_{C}\right)$

The Grid Inverter. In the grid inverter created by transistors $T 1-T 4$, transistors $T 1$ and $T 2$ clamp grid neutral point to the DC link terminals with $50 \%$ duty cycle $D$, this allows simultaneous operation of both: generator converter and grid inverter; but requires two times greater DC link voltage. The $U_{C m}$ voltage magnitude is set at nominal grid voltage. The phase angle $\delta$ is calculated in the DC link voltage control loop using $P I$ and $G_{1}$ blocks. The $P I$ controller is tuned to suppress energy oscillations and the $G_{1}$ is a zero order transfer function. The phase locked loop PLL block is used to displace inverter's output voltage $u_{C}$ in relation to line voltage $u_{S}$. Figure 7 shows control algorithm for grid side inverter.

Properties of the proposed interconnection solution have been investigated experimentally during $P_{G}$ customer side generator power changes in the situation of power consumed by the load $P_{L}$ greater than power produced by customer side generator $P_{G}$, (see Fig. 8). As one can see the proposed arrangement secures the power balance at the point of common coupling. Before and after changes all the power balancing rules are satisfied, load voltage magnitude is stable and line current changes are of aperiodic type. Also some results concerning the situation of supply voltage interruptions are presented (Fig. 9). During supply voltage interruptions the considered system secures constant power delivered to the load and, what is the most important, without worsening the voltage quality.

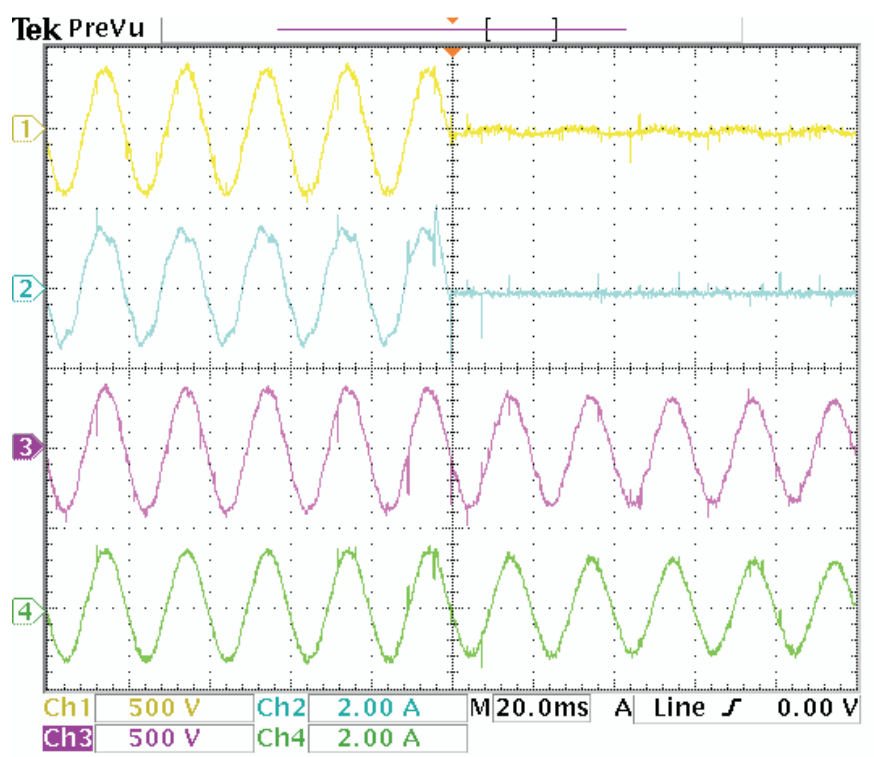

Fig. 9. Experimental results during $u_{S}$ interruptions ( $D C$ customer side generator); $\left(\mathrm{Ch} 1-u_{S}, \mathrm{Ch} 2-i_{S}, \mathrm{Ch} 3-u_{C}, \mathrm{Ch} 4-i_{L}\right)$

Summarizing, the proposed solution has capabilities to realize the following ancillary services to grids: current and voltage harmonics compensation; reactive power compensation; load voltage stabilization; balancing the network in conditions of unbalanced loads; uninterruptible supplying. All the latter mentioned functions are available without any system reconfigurations and can be realized simultaneously.

\section{Conclusions}

The aim of this paper has been to present the fundamental problems of the Electrical Power Systems and the efficiency 
improvement services to grids which are possible with Distributed Generation - it is envisaged that as alternatives to conventional solutions (new power plants, new transmission lines, substations, and associated equipment) the new players can provide the same services to the grids.

The paper has also introduced a system for the utilization of the customer-side generated energy - an arrangement was built on the basis of a Voltage Active Power Filter. The proposed system, assigned for customer-side generation interconnection secures also some improvement services to grids: these services include: current active filtering, load voltage stabilization and in case of grid voltage faults - uninterruptible load supplying (possible with energy storage device). The experimental results, presented in the paper confirm the flexibility of this arrangement in processes of power delivery improvement. Because of its simple structure, uncomplicated control strategy and the number of functions possible to realize, this solution seems to be very suitable for end-users connected to the weak grids.

\section{REFERENCES}

[1] P. Kundur, Power System Stability and Control, McGraw-Hill, New York, 1994.

[2] J. Machowski, J. Bialek, and J. Bumby, Power System Dynamics and Stability, John Wiley\&Sons, New York, 1997.

[3] N. Hingorani and L. Gyugyi, Understanding FACTS: Concepts and Technology of Flexible AC Transmission Systems, IEEE, New York, 2000

[4] A. Ghosh and G. Ledwich, Power Quality Enhancement Using Custom Power Devices, Kluwer Academic Publishers, Boston, 2002.
[5] R. Dugan, M. Mc Granaghan, and W. Beaty, Electrical Power Systems Quality, McGraw-Hill, New York, 1996.

[6] J. Arrillaga, N. Watson, and S. Chan, Power System Quality Assessment, John Wiley\&Sons, Chichester, 2000.

[7] T. Kurowski, G. Benysek, A. Kempski, and R. Smolenski, About Proper Cooperation of The Electric Drives and Static Converters, Wroclaw University of Technology Press, Wroclaw, 2000, (in Polish).

[8] P. Thomsen, Application and Control of CUPS in the Distribution Grid, Institute of Energy Technology, Aalborg, 1999.

[9] R. Strzelecki, G. Benysek, and M. Jarnut, "Power quality conditioners with minimum number of current sensors requirement”, Electrotechnical Review 11, 295-298 (2008).

[10] R. Strzelecki, H. Tunia, M. Jarnut, G. Meckien, and G. Benysek, "Transformerless 1-phase active power line conditioners", Proc. $34^{\text {th }}$ IEEE Power Electronics Specialist Conf. 1, 321326 (2003).

[11] G. Benysek, Improvement in the Quality of Delivery of Electrical Energy Using Power Electronics Systems, Springer-Verlag, London, 2007.

[12] R. Strzelecki and G. Benysek, Power Electronics in Smart Electrical Energy Networks, Springer-Verlag, London, 2008.

[13] Z. Chen, X. Zhang, and J. Pan, "An integrated inverter for a single-phase single-stage grid-connected PV system based on Z-source", Bull. Pol. Ac.: Tech. 55 (3), 263-272 (2007).

[14] Y. Jiang, Z. Chen, J. Pan, X.I Zhao, and P. Lee, "A novel phase-shift full-bridge converter with voltage-doubler and decoupling integrated magnetics in PV system", Bull. Pol. Ac.: Tech. 56 (3), 285-293 (2008).

[15] R. Strzelecki, G. Benysek and M. Jarnut, "Interconnection of the customer-side resources using single phase VAPF", Electrotechnical Review 10, 59-65 (2007). 